\title{
Nicotinamide-functionalized carbon quantum dot as new sensing platform for portable quantification of vitamin B12 in fluorescence, UV-Vis and smartphone triple mode
}

\section{Sahar Dadkhah}

KN Toosi: KN Toosi University of Technology

Ali Mehdinia ( $\square$ mehdi_3848@yahoo.com )

Iranian National Institute for Oceanography and Atmospheric Science https://orcid.org/0000-00016080-0681

\section{Ali Jabbari}

KN Toosi: KN Toosi University of Technology

\section{Ahmad Manbohi}

Iranian National Institute for Oceanography and Atmospheric Science

\section{Research Article}

Keywords: Fluorescence, smartphone-assisted, colorimetric nanosensor, vitamin B12, nicotinamidefunctionalized CQDs, supplements

Posted Date: July 16th, 2021

DOI: https://doi.org/10.21203/rs.3.rs-683491/v1

License: (c) (i) This work is licensed under a Creative Commons Attribution 4.0 International License. Read Full License

Version of Record: A version of this preprint was published at Journal of Fluorescence on January 18th, 2022. See the published version at https://doi.org/10.1007/s10895-021-02863-5. 


\section{Abstract}

Development of an efficient, portable and simple nanosensor-based systems with reliable analytical performance for on-site monitoring of vitamin B12 (VB12) are still major problems and a challenging work for quality control of manufacturers. Herein, a new fluorescence, UV-Vis and smartphone triple mode nanosensors were designed for the simultaneous detection of VB12 with high sensitivity and accuracy. A novel nanosensor was synthesized through nicotinamide-functionalizing on carbon quantum dot (NACQDs) by an one-step microwave-assisted method with green approaches. The NA-CQDs as new nanosensor showed excellent fluorescence properties and wide linear ranges from 0.1-60 $\mu \mathrm{M}$ with the detection limits of $31.7 \mathrm{nM}$. Moreover, color changes of NA-CQDs induced by the VB12 could also be detected by UV-Vis spectrophotometer and inhouse-developed application installed on smartphone as a signal reader, simultanusly. The Red, Green and Blue (RGB) intensities of the colorimetric images of NACQDs/VB12 system which taken by smartphone's camera converted into quantitative values by the introduced application. A smartphone-integrated with NA-CQDs as colorimetric sensing platform displays good linear ranges (4.16 to $66.6 \mu \mathrm{M})$ for on-site determination of VB12 with detection limit of $1.40 \mu \mathrm{M}$. The method was successfully applied in the determination of VB12 in complex pharmaceutical supplement formulations without any sample pre-treatment and matrix interfering effects. The recovery results (96.52-105.10\%) which are in agreement with the reference methods, demonstrating the capability of the smartphone-assisted colorimetric sensing platform in many on-site practical applications of quality controls.

\section{Introduction}

Vitamin B12 (VB12), also called as cyanocobalamin, plays a vital role in normal metabolism of enzymes, lipids and carbohydrates, nerve cell maintenance, DNA synthesis and red blood cell formation [1-4]. For adults, the recommended dietary allowance of VB12 are about $0.40-2.80 \mathrm{mg}$ per day [5]. The excessive VB12 can resulted in liver disease, asthma, neurotoxicity and kidney disorders. Moreover, deficiency of VB12 may lead to significant public health problems like neurological degeneration, memory loss, pernicious anemia and increases the risk of heart disease [6]. Vitamin B12 is one of the essential water soluble "B complex vitamins" that cannot be produced by body cells, it can also be taken from foods like milk, meat, egg and pharmaceutical supplements in various dosage forms daily [7]. As recommended by a healthcare practitioner, the deficiency of VB12 can often be reversed by consuming supplements or by fortified foods [8]. At present there is great attention in accurately assessing the total dietary intake of vitamins B12 from all sources, especially vitamin supplements (tablets or injections). Therefore, there is an urgent need to develop an accurate, fast and portable method for on-site quantification of VB12 at the quality control of pharmaceutical companies.

At present, most quantification of vitamin B12 are conducted using high performance liquid chromatography (HPLC) [9], enzyme-linked immunosorbent assay (ELISA) [10], chemiluminescence [11], liquid chromatography/electrospray ionization-tandem mass spectrometry (LC/ESI-MS/MS) [12], capillary electrophoresis and electrochemical methods [7, 13]. Generally, these laboratory-based methods 
are expensive both in reagent and services and requires professionally trained operators [14]. Also, another limitation of laboratory-based methods is their time-consuming properties, which leads to factory production line wait for several hours for the test results in order to release the high-quality products. Therefore, the development of an efficient, affordable and simple nanosensor-based systems with sufficient analytical performance for accurate and fast monitoring of vitamin B12 content is necessary for the evaluation of supplement quality.

With the progress of quantum dots as advanced nonomaterials, they are considered as promising fluorescence sensing platform for quantification of VB12 $[15,16]$. Compared with conventional semiconductor quantum dots, carbon-based quantum dots (CQDs) offer benefits such as highly tunable photoluminescence, chemical inertness, small size and photo-bleaching resistance and also low toxicity and biocompatibility $[17,18]$. Up to now, among developed nanosensor for quantitative detection of VB12, fluorescence-based nanosensor using quantum dots have been reported more than the others $[5,8$, 19]. It should be noted that, most of the developed fluorescent assays of VB12 for many applications are still conducted with expensive instruments in laboratory and are not suitable for fast on-site detection of analytes.

In recent years, smartphone-based detection system integrated with bio/chemical sensors has shown promising progress in point of care (POC)/ Point of use (POU) testing approaches for various analytes. Because they are portable, ubiquitous and also produce rapid and simple quantitative results that can be interpreted by untrained personnel especially in resource-limited areas [14, 20-23]. To-date, one research teams have been reported smartphone platform for POC quantification of VB12 in blood using immonuassay-based lateral flow biosensors [24]. These biosensors fabrication needs complicated antibody preparation technology and test strip assembly skill, which is not easy to achieve. Furthermore, in the present study, a novel nicotinamide-functionalized CQDs nanosensor were designed as fluorescence and colorimetric probes for simple and portable detection of VB12 simultaneously. NA-CQDs were synthesized by one-pot microwave-assisted hydrothermal method emploing nicotinamide as new sources of functionalized material on carbon quantum dots for the first time. Moreover, we have try to make new nanosensor more user friendly and portable by developing in-house android-app on smartphone. But to our knowledge, no such work has been reported so far by the smartphone integrated with NA-CQDs nanosensors for the on-site monitoring of VB12 in pharmaceutical supplements. The NACQDs nanosensor exhibited dual response via both fluorescence and colorimetric change for on-site monitoring of VB12, simultaneously. In the RGB-based (red, green and blue color space) software, while the image is captured by the camera, simultaneous analysis can be done by the in-house developed android app and the quantitative results are displayed on the smartphone. Therefore, the portable smartphone-assisted colorimetric reader is promising to become a sensitive tool for quantitative colorimetric measurement and accurate on-site analysis of VB12 in many applications.

\section{Experimental}

\subsection{Materials and Instruments}


All standards or chemical reagents used were of pharmaceutical and analytical grades. Nicotinamide, pyridoxine hydrochloride (vitamin B6), thiamine hydrochloride (vitamin B1), calcium D-pantothenate (vitamin B5), and sodium hydroxide were purchased from Merck (Darmstadt, Germany) and cyanocobalamin (vitamin B12) and citric acid (CA) were supplied from Sigma-Aldrich.

Ultrapure water was purified with a Millipore $\left(0.05 \mu \mathrm{S} \mathrm{cm}^{-1}\right)$ instrument. All UV-Vis absorption spectra were measured by a UV-Vis spectrophotometer (Cary 100 UV-Vis, Agilent Technologies) from 200 to 800 $\mathrm{nm}$. Fluorescence spectra of VB12 standard and sample solutions were recorded from 370 to $700 \mathrm{~nm}$ by a fluorescence spectrophotometer (LS-55, Perkin Elmer) equipped with a Xenon lamp source and a $1.0 \mathrm{~cm}$ quartz cell. Surface morphology microscopy of NA-CQDs were performed using a SEM (ZEISS SIGMA VP FE-SEM with Oxford EDS and Mapping) and high-resolution TEM (FEI Tecnai F20 HR-TEM) microscope. The FT-IR spectrum of NA-CQDs were acquired using a Fourier transform infrared spectrometer (FT-IR Spectrum two, Perkin Elmer) from 400 to $4000 \mathrm{~cm}^{-1}$.

\subsection{Microwave-assissted synthesis of nicotinamide- functionalized CQDs}

Nicotinamide-functionalized CQDs have been successfully synthesized via a facile hydrothermal treatment of citric acid as $\mathrm{C}$ source and nicotinamide as new source of $\mathrm{N}$ through microwave-assisted hydrothermal method. In a typical experiment citric acid monohydrate $(1.68 \mathrm{~g})$ and nicotinamide $(2.92 \mathrm{~g})$ were thoroughly dissolved in $10 \mathrm{~mL}$ of ultrapure water and stirred for $30 \mathrm{~min}$. Then the solution was transferred into microwave radiation system with moderate temperature $\left(80 \mathrm{~W}, 160^{\circ} \mathrm{C}\right)$ for $20 \mathrm{~min}$.

\subsection{Development of RGB-based smartphone-app}

In smartphone-assisted colorimetric sensing platform, the color intensities of the images taken by the camera are proportional to the red, green, and blue (RGB) values. For on-site image processing, in-house android-based app was developed by an algorithm similar to ImageJ software (U.S. National Institutes of Health, Bethesda, MD, USA) for quantifying color intensities and relating it to VB12 concentration. In this study, all images were taken without a flash with a 25-megapixel camera of Samsung smartphone which that positioned as close as possible to the sample container (about $6 \mathrm{~cm}$ away). The RGB intensities of the colorimetric images of NA-CQDs/VB12 system which taken by smartphone's camera converted into quantitative values by the introduced application.

\subsection{Detection procedure of VB12 and real sample preparation}

Initially, A stock standard solution of VB12 with $1 \mathrm{mM}$ concentration were prepared. The different concentrations of VB12 solution were prepared by appropriate dilution of stock standard by ultrapure water. The NA-CQDs nanosensor were used to determination of VB12 by the following procedure. $1 \mathrm{ml}$ of NA-CQDs solution $(0.1 \mathrm{mg} / \mathrm{ml})$ was added to $5 \mathrm{ml}$ tube. Then $3.5 \mathrm{ml}$ of disodium hydrogen phosphate buffer solution $(10 \mathrm{mM}, \mathrm{pH} 7)$ and $500 \mu \mathrm{l}$ of VB12 solution with different concentrations was added to the above mixture solution. Fluorescence and UV-Vis spectra of NA-CQDs/VB12 system were recorded from 
370 to $700 \mathrm{~nm}$ at excitation wavelength of $365 \mathrm{~nm}$ and from 200 to $800 \mathrm{~nm}$ by the fluorescence and UVvis spectrophotometer, respectively. Also the color change of NA-CQDs solution-based nanosensor upon addition different concentrations of VB12 were scanned using the smartphone camera through RGB application, simultaneously.

In order to real sample evaluation, the VB12 injection and tablet were purchased from a local pharmacy. 1 $\mathrm{ml}$ of VB12 injection containing, $333.3 \mu \mathrm{g}$ VB12 per $\mathrm{ml}$, was added to a $10 \mathrm{ml}$ volumetric flask and diluted with ultrapure water to prepare solution with concentration of $24.593 \mu \mathrm{M}$. For VB12 tablet sample preparation, containing $200 \mu \mathrm{g}$ VB12 per tablet, accurately weighted powder equivalent 1 tablet and dissolved with $10 \mathrm{ml}$ of ultrapure water into volumetric flask (concentration of VB12 solution is 14.756 $\mu \mathrm{M})$. Then, the fluorescence, UV-Vis spectra and color intensities of the real samples were recorded according the above-mentioned procedure.

\section{Results And Discussion}

\subsection{Characterization of nicotinamide-functionalized CQDs 3.1.1 FT-IR}

The surface chemical groups of nicotinamide-functionalized CQDs were further characterized by FT-IR (Fig. 1A). For NA-CQDs, a broad absorption band was observed at $3000-3400 \mathrm{~cm}^{-1}$, which is attributed to stretching vibrations of $\mathrm{O}-\mathrm{H}$ and $\mathrm{N}-\mathrm{H}$ groups. Moreover FT-IR spectrum of NA-CQDs exhibited characteristic absorption peaks at around 1659 and $1700 \mathrm{~cm}^{-1}$ which are belong to $\mathrm{C}=\mathrm{C}$ and $\mathrm{C}=\mathrm{O}$ stretching vibrations bands. Furthermore, nicotinamide had been successfully functionalized on the carbon quantum dots due to the appearance of bending vibration of $\mathrm{C}-\mathrm{NH}$ and stretching vibration of C$\mathrm{N}$ at 1380 and $1550 \mathrm{~cm}^{-1}$, respectively $[4,25]$. The results obtained from FT-IR are in agreement with other characterization and clearly confirm that nicotinamide-functionalized CQDs are successfully synthesized.

\subsubsection{SEM and EDX elemental mapping}

The morphology and elemental composition of NA-CQDs were characterized by FE-SEM apparatus which was equipped with an energy-dispersive X-ray (EDX) spectroscope. Figure 1B and Fig. 1D exhibit the FESEM images of the NA-CQDs in different magnifications to evaluate their morphology and internal structure. The figures reveal that the synthesized NA-CQDs were all spherical in shape and fairly uniform in size.

EDX analysis and mapping were also conducted to evaluate the detailed elemental composition and distribution. The data generated from EDX spectrum of NA-CQDs showed the following atomic percentages, respectively: $67.8 \mathrm{wt} \%(\mathrm{C}), 11.1 \mathrm{wt} \%(0)$ and $21.1 \mathrm{wt} \%(\mathrm{~N})$. In addition EDX mapping in the 
Fig. 1C, confirmed that successful and uniform excistence of $\mathrm{N}$ element in the structure of nicotinamidefunctionalized CQDs.

\subsubsection{HR-TEM}

Morphological and size distribution of NA-CQDs were further obtained by high-resolution transmission electron microscopy (HR-TEM). HR-TEM image (Fig. 1E) illustrate that, the average diameter of NA-CQDs was smaller than $10 \mathrm{~nm}$.

\subsection{Fluorescence sensing performance of NA-CQDs for VB12}

The capability of NA-CQDs as a fluorescence nanoprobe for monitoring of VB12 was investigated. Figure. $2 \mathrm{~A}$ shows that as the concentration of VB12 increases, the fluorescence emission intensity of NA-CQDs is clearly decreased. Under the optimal conditions (Fig. S1), a good linear relationship of $F / F_{0}$ with concentration of VB12 was obtained in the range of 0.1-60 $\mu \mathrm{M}$ (Fig. 2B). Moreover, the linear regression was fitted based on Stern-Volmer equation $\left(F / F_{0}=0.0294\right.$ CB12 + 0.9641) with good correlation coefficients (0.9975). The limit of detection (LOD) was found to be $31.7 \mathrm{nM}$ (based on the Eq. 3SD/m, where SD and $m$ referred to standard deviation of the blank and the slope of the calibration curve, respectively. Compared with other probes based on fluorescence nanosensor for detection of VB12, the synthesized NA-CQDs nanosensor exhibit wide linear range, high sensitivity and low detection limit, which is lower than these of the recent reports (Table.1).

\subsection{Smartphone-assisted colorimetric sensing platform for VB12}

Taking advantage of good performance of smartphone-assissted colorimetric platform for sensing analytes, the NA-CQDs/ VB12 system was utilized for on-site monitoring of VB12. As shown in the Fig. 3, the color change of the NA-CQDs probe from blue to red induced by VB12, which were quantified through RGB profiling in the range of 4.16-66.6 $\mu \mathrm{M}$. As revealed in Fig. 3, a good linear regression equation $\left(R^{2}=\right.$ 0.9959) between $R /(R+G+B)$ intensities with respect to VB12 concentration was obtained $(R /(R+G+B)$ $\left.=0.0041 \mathrm{C}_{\mathrm{B} 12}+0.2949\right)$.

The color change of NA-CQDs nanosensor upon addition different concentrations of VB12 can be observed by naked eyes under natural light. The colorimetric signal of NA-CQDs/VB12 system were captured by the smartphone camera and analyzed by in-house android-app for the total start-to-results time of less than $5 \mathrm{~min}$. The fast and on-site results of smartphone-based sensor demonstrating the capability of smartphone-assisted colorimetric sensing platform for quality control of pharmaceutical products.

\subsection{Mechanism investigation of fluorescence quenching and color change of NA-CQDs nanosensor}


The fluorescence of NA-CQDs was effectively quenched in the presence of different concentrations of vitamin B12. For the mechanism of VB12-induced fluorescence quenching of NA-CQDs, the reasonable explanation was that, IFE-based fluorescent quenching mechanism. As illustrated in Fig. 4A, the absorption spectral overlap of VB12 with the fluorescence excitation and emission peak of nicotinamidefunctionalized CQDs, meets the requirement for inner filter effect fluorescence quenching strategy for sensing of VB12. This reasonable assumption does fit well with the previous literature $[18,26]$.

In the present study, to more clarify color change in the NA-CQDs/B12 system, the UV-visible absorption spectra of N-CQDs in the absence and presence of VB12 were investigated and the results illustrated in the Fig. 4B. As shown in Fig. In the absence of VB12, the NA-CQDs had a characteristic peak about 334 $\mathrm{nm}$. In the presence of NA-CQDs, no shift in wavelength was observed in the absorption spectra of VB12 $(33 \mu \mathrm{g} / \mathrm{ml})$ but the peak at about $360 \mathrm{~nm}$ increases after additions of VB12 (Fig. 4B). The addition of VB12 (0-133.2 $\mu \mathrm{M})$ into the NA-CQDs solution gradually increases the intensity of absorption band at about $360 \mathrm{~nm}$, which resulted in color change of NA-CQDs nanosensor (Fig. 4C).

\subsection{Selectivity and interference studies for the detection of VB12}

Various common substances in VB12 supplements formulation may interfere with the accuracy of B12 detection. Therefore, Vitamin B1, B5 and vitamin B6 were applied as competitive vitamins for selectivity studies of the NA-CQDs nanosensor in various supplements. To evaluate the selectivity of the NA-CQDs nanosensor, VB12 was detected in the presence of other competitive vitamins including vitamin B1, B5 and vitamin B6. Figure 5A shows the quenching effect of the NA-CQDs/VB12 (B12, $30 \mu \mathrm{M})$ system in the in the presence of vitamin $B 1(B 1,3000 \mu M), B 5(B 5,3000 \mu M)$ and vitamin $B 6(B 63000 \mu M)$. The results indicated that, even 100 -fold concentrations of competitive vitamins have no obvious effect on the B12 detection.

To further confirm the IFE quenching mechanism and the selectivity of NA-CQDs nanosensor, several UVvis absorption spectra of different vitamins, including vitamin B1, B5 and vitamin B6 and B12 are also displayed in Fig. S5. It is obvious that only the UV-vis absorption spectrum of VB12 have the significant overlap with the excitation $(366 \mathrm{~nm})$ and emission spectrum $(444 \mathrm{~nm})$ of NA-CQDs.

Neurobion is a brand of supplements that contain a combination of B-complex vitamins. Based on summary of product characteristics of European medicine agency guidelines for Neurobion injection and Tablet, possible interferes which related to excipients of different pharmaceutical formulations, were also evaluated. Moreover, As shown in Fig. 5B, it was obvious that these coexisting excipients did not interfere with the detection of vitamin B12 (Fluorescence change $<2 \%$ ). Therefore, the results demonstrate that the selectivity of nanosensor toward VB12 and the possibility of practical application of NA-CQDs nanosensor in the determination of VB12 in various pharmaceutical supplements formulation (injection and tablet).

\subsection{Real samples evaluation}


The applicability of the NA-CQDs nanosensors for quantitative detection of B12 were evaluated in pharmaceutical VB12 supplements in various dosage forms (injection and tablet) as real samples.

Table. 2 indicates the results obtained by the fluorescence, UV-Vis and smartphone-integrated colorimetric sensing platform for the on-site quantification of VB12 in the B12 injection and tablet, respectively. The recovery values of VB12 by the introduced NA-CQDs nanosensors in the real samples are in the ranges 96.52-105.10\% with RSD between $1.32 \%$ and $3.44 \%(n=3)$. These results were compared to their label/manufacture's claims with the reference method (USP 42 vitamin oral solution for analysis of VB12) and a high level of agreement was found (HPLC chromatograms Fig.S2 Fig.S3 Fig.S4).

\section{Conclusion}

We have successfully designed a triple mode detection of B12 via novel nicotinamide-functionalized CQDs nanosensor with benefit of advantages of universal fluorescence and UV-Vis methods and smartphone-assisted colorimetric sensing platform as new generation of analytical tools for bio/chemical detections. The satisfied recovery results (96.52-105.10\%) which are in agreement with the reference method in various dosage forms of VB12 supplements like an injection and tablet, demonstrating that the capability of new sensing platform for on-site monitoring in quality control of pharmaceutical manufactures. Considering excellent optical properties of NA-CQDs, high sensitivity, good selectivity, rapid response time, portability, wide linear range, simplified data analysis/interpreting by the proposed method toward VB12 detection, it is believed that, besides the promising fluorescence sensing performance of NA-CQDs, applied smartphone-assisted colorimetric sensing platform for quantifying of color change induced by the VB12 could also afford experience for designing neotype sensing strategy for point of care (POC)/ Point of use (POU) testing in many practical applications.

\section{Declarations}

\section{Author Declarations}

-Funding: Not applicable

-Conflicts of interest: The authors declare that there is no conflict of interest.

-Ethics approval/declarations: Not applicable

-Consent to participate: Not applicable

-Consent for publication: Not applicable

-Availability of data and material/ Data availability: Authors confirm that all relevant data are included in the article and/or its supplementary information files

-Code availability: Not applicable 
-Authors' contributions: Sahar Dadkhah carried out the experiments. Sahar Dadkhah wrote the initial format of the manuscript with support from Ali Mehdinia. Ahmad Manbohi and Ali Jabbari helped supervise the project. Ali Mehdinia supervised the project.

\section{References}

1. Li Y, Jia Y, Zeng Q, Jiang X, Chengs Z (2019) A multifunctional sensor for selective and sensitive detection of vitamin B12 and tartrazine by Förster resonance energy transfer. Spectrochim Acta A 211:178-188

2. Ravi PV, Thangadurai TD, Natarajs D (2020) Ultra-sensitive detection of commercial vitamin $B 9$ and B12 by graphene nanobuds through inner filter effect. J. Photochem. Photobiol. A: 112691

3. Chakravarty S, Gogoi B, Mandal BB, Bhardwaj N, Sarmas NS (2018) Silk fibroin as a platform for dual sensing of vitamin B12 using photoluminescence and electrical techniques. Biosens Bioelectron $112: 18-22$

4. Sun Z, Chen Z, Luo J, Zhu Z, Zhang X, Liu R, and Z.-c. Wus (2020) A yellow-emitting nitrogen-doped carbon dots for sensing of vitamin B12 and their cell-imaging. Dyes Pigm. 176: 108227

5. Du F, Cheng Z, Kremer M, Liu Y, Wang X, Shuang S, Dongs C (2020) A label-free multifunctional nanosensor based on N-doped carbon nanodots for vitamin B 12 and Co $2+$ detection, and bioimaging in living cells and zebrafish. J. Mater. Chem. B

6. Jia Y, Hu Y, Li Y, Zeng Q, Jiang X, Chengs Z (2019) Boron doped carbon dots as a multifunctional fluorescent probe for sorbate and vitamin B12. Microchim Acta 186(2):1-10

7. Li Y, Gill BD, Grainger MN, Manley-Harriss M (2019) The analysis of vitamin B12 in milk and infant formula: A review. Int Dairy J 99:104543

8. Gore AH, Kale MB, Anbhule PV, Patil SR, Kolekars GB (2014) A novel FRET probe for selective and sensitive determination of vitamin $B 12$ by functionalized CdS QDs in aqueous media: applications to pharmaceutical and biomedical analysis. RSC Advances 4(2):683-692

9. Qiu X, Zhang H, Yin Y, Brandes H, Marsala T, Stenerson K, Cramer H, Yous H (2019) Determination of active vitamin B12 (cobalamin) in dietary supplements and ingredients by reversed-phase liquid chromatography: Single-laboratory validation. Food Chem 298:125010

10. Kong D, Liu L, Song S, Kuang H, Xus C (2017) Development of sensitive, rapid, and effective immunoassays for the detection of vitamin B 12 in fortified food and nutritional supplements. Food Anal Methods 10(1):10-18

11. Lok KS, Lee PPF, Kwok YC, Nguyens N-T (2012) Rapid determination of vitamin B 12 concentration with a chemiluminescence lab on a chip. Lab Chip 12(13):2353-2361

12. Schwertner HA, Valtier S, Bebartas VS (2012) Liquid chromatographic mass spectrometric (LC/MS/MS) determination of plasma hydroxocobalamin and cyanocobalamin concentrations after hydroxocobalamin antidote treatment for cyanide poisoning. J Chromatogr B 905:10-16 
13. Dong S, Chi L, He P, Wang Q, Fangs Y (2009) Simultaneous determination of antioxidants at a chemically modified electrode with vitamin B12 by capillary zone electrophoresis coupled with amperometric detection. Talanta 80(2):809-814

14. Aguirre M, Long KD, Canals A, Cunninghams BT (2019) Point-of-use detection of ascorbic acid using a spectrometric smartphone-based system. Food Chem 272:141-147

15. Vaishnavi E, Renganathans R (2013) CdTe quantum dot as a fluorescence probe for vitamin B12 in dosage form. Spectrochim Acta A 115:603-609

16. Wang J, Wei J, Su S, Qius J (2015) Novel fluorescence resonance energy transfer optical sensors for vitamin B 12 detection using thermally reduced carbon dots. New J Chem 39(1):501-507

17. Gong X, Zhang Q, Gao Y, Shuang S, Choi MM, Dongs C (2016) Phosphorus and nitrogen dual-doped hollow carbon dot as a nanocarrier for doxorubicin delivery and biological imaging. ACS Appl Mater Interfaces 8(18):11288-11297

18. Zhang L, Wang H, Hu Q, Guo X, Li L, Shuang S, Gong X, Dongs C (2019) Carbon quantum dots doped with phosphorus and nitrogen are a viable fluorescent nanoprobe for determination and cellular imaging of vitamin B 12 and cobalt (II). Microchim Acta 186(8):506

19. Ding L, Yang H, Ge S, Yus J (2018) Fluorescent carbon dots nanosensor for label-free determination of vitamin B12 based on inner filter effect. Spectrochim Acta A 193:305-309

20. Zhang S, Tis TB, Wei Q (2020) Smartphone-based clinical diagnostics. In: Precision Medicine for Investigators, Practitioners and Providers. Elsevier, pp 493-508

21. Wang T, Mei Q, Tao Z, Wu H, Zhao M, Wang S, Lius Y (2020) A smartphone-integrated ratiometric fluorescence sensing platform for visual and quantitative point-of-care testing of tetracycline. Biosens Bioelectron 148:111791

22. You X, Huang C, Luo Y, Shi G, Zhou T, Dengs J (2020) A smartphone-based platform for point-of-use determination of alkaline phosphatase as an indicator of water eutrophication. Microchim Acta 187(6):1-10

23. Liu J, Geng Z, Fan Z, Liu J, Chens H (2019) Point-of-care testing based on smartphone: The current state-of-the-art (2017-2018). Biosens Bioelectron 132:17-37

24. Lee S, O'Dell D, Hohenstein J, Colt S, Mehta S, Ericksons D (2016) NutriPhone: a mobile platform for low-cost point-of-care quantification of vitamin B 12 concentrations. Sci Rep 6:28237

25. Zhang H, Chen Y, Liang M, Xu L, Qi S, Chen H, Chens X (2014) Solid-phase synthesis of highly fluorescent nitrogen-doped carbon dots for sensitive and selective probing ferric ions in living cells. Anal Chem 86(19):9846-9852

26. Zu F, Yan F, Bai Z, Xu J, Wang Y, Huang Y, Zhous X (2017) The quenching of the fluorescence of carbon dots: a review on mechanisms and applications. Microchim Acta 184(7):1899-1914

27. Yu Y, Li C, Chen C, Huang H, Liang C, Lou Y, Chen X-B, Shi Z, Feng S (2019) Saccharomyces-derived carbon dots for biosensing $\mathrm{pH}$ and vitamin B 12. Talanta 195:117-126 


\section{Tables}

Table. 1 Comparison of different quantum dots fluorescence based probes for the detection of VB12

\begin{tabular}{|c|c|c|c|c|}
\hline FL Probe & Linear range & Detection limit & References & \\
\hline CDs & $0.5-60 \mu \mathrm{M}$ & $0.1 \mu \mathrm{M}$ & [19] & Carbon dots: CDs \\
\hline (MPA) functionalized CdS QDs & 3.69-73.78 $\mu \mathrm{M}$ & $5.10 \mu \mathrm{M}$ & [8] & Mercaptopropionic \\
\hline Y-CDs & $5-200 \mu \mathrm{M}$ & $2.045 \mu \mathrm{M}$ & [4] & acid functionalized \\
\hline $\mathrm{t}-\mathrm{CD}$ & $0.74-8.85 \mu \mathrm{M}$ & $0.073 \mu \mathrm{M}$ & [16] & $\begin{array}{l}\text { CdS quantum dots } \\
\text { (MPA) }\end{array}$ \\
\hline CdTe QDs & $0.74-10.33 \mu \mathrm{M}$ & $0.110 \mu \mathrm{M}$ & [15] & functionalized CdS \\
\hline $\mathrm{s}-\mathrm{N}-\mathrm{CDs}$ & $10-100 \mu \mathrm{M}$ & $2.19 \mu \mathrm{M}$ & [27] & QDs \\
\hline $\mathrm{N}$-CNDs & $0.5-35 \mu \mathrm{M}$ & $47.4 \mathrm{nM}$ & [5] & $\begin{array}{l}\text { Yellow luminescent } \\
\text { carbon dots: Y-CDs }\end{array}$ \\
\hline NA-CQDs & $0.1-60 \mu \mathrm{M}$ & $31.7 \mathrm{nM}$ & This work & $\begin{array}{l}\text { Thermally-reduced } \\
\text { carbon dot: t-CD }\end{array}$ \\
\hline
\end{tabular}

CdTe QDs

saccharomyces-derived N-doped carbon dots: S-N-CDs

N-doped carbon nanodots: N-CNDs

Nicotinamide-functionalized CQDs: NA-CQDs

Table.2 Analytical results for the detection of VB12 in real samples $(n=3)$

\section{Figures}




\begin{tabular}{|llllll|}
\hline & Method & $\begin{array}{l}\text { Claimed content } \\
(\mu \mathrm{M})\end{array}$ & Found $(\mu \mathrm{M})$ & $\begin{array}{l}\text { RSD }(\%, \\
\mathbf{n}=3)\end{array}$ & $\begin{array}{l}\text { Recovery } \\
(\%)\end{array}$ \\
\hline \multirow{2}{*}{$\begin{array}{l}\text { VB12 } \\
\text { Injection }\end{array}$} & Fluorescence & 24.593 & 25.297 & 1.32 & 102.86 \\
\cline { 2 - 6 } & Smartphone & 24.593 & 25.848 & 2.86 & 105.10 \\
\cline { 2 - 6 } & UV-Vis & 24.593 & 24.922 & 1.63 & 101.33 \\
\hline VB12 Tablet & Fluorescence & 14.756 & 14.510 & 1.66 & 98.33 \\
& Smartphone & 14.756 & 14.243 & 3.44 & 96.52 \\
\cline { 2 - 6 } & UV-Vis & 14.756 & 14.473 & 2.63 & 98.08 \\
\hline
\end{tabular}



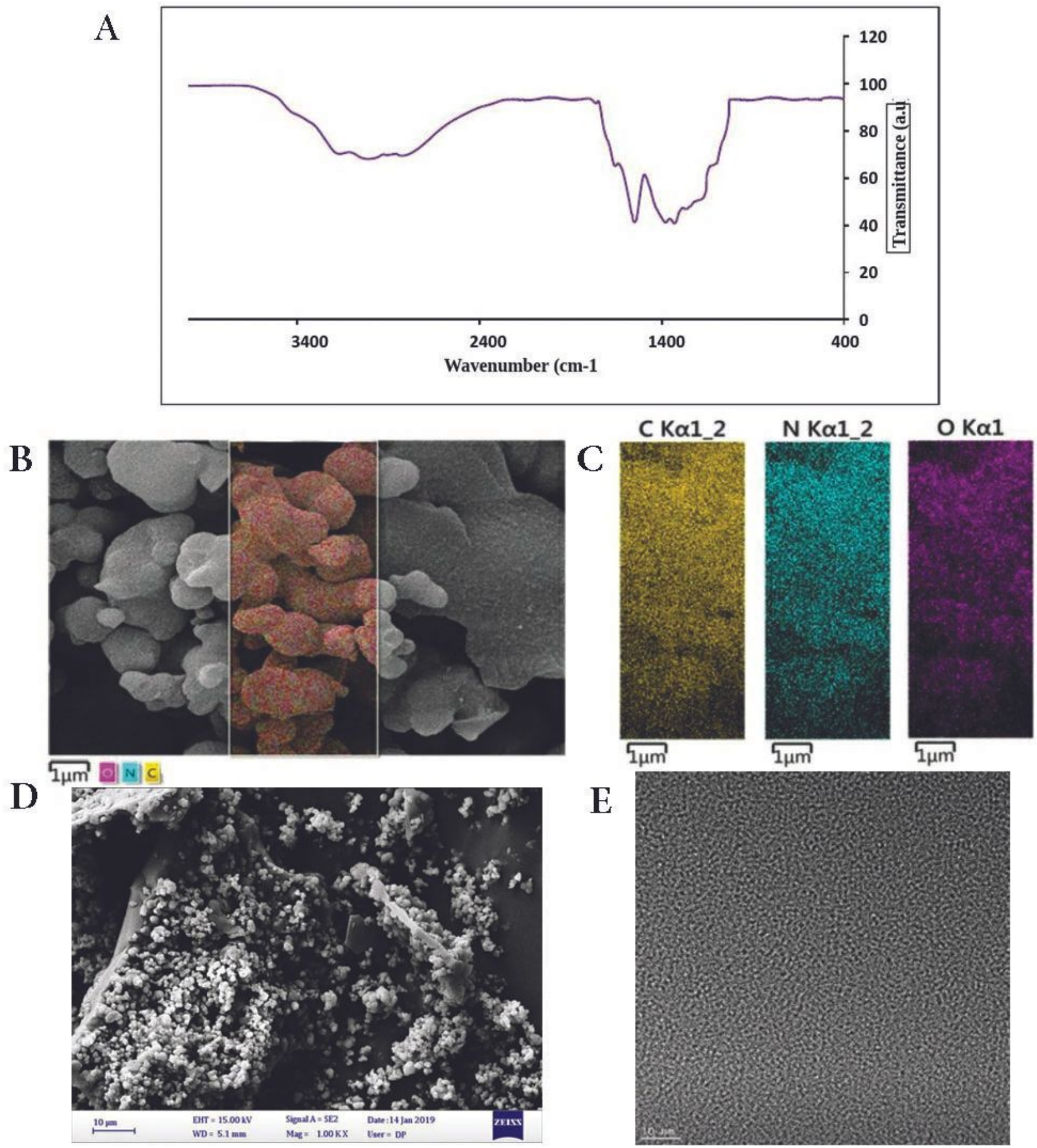

\section{Figure 1}

(A) FT-IR spectra, $(B, D)$ FE-SEM images of NA-CQDs with different magnification. (C) EDX elemental mapping and (E) HR-TEM image of NA-CQDs 

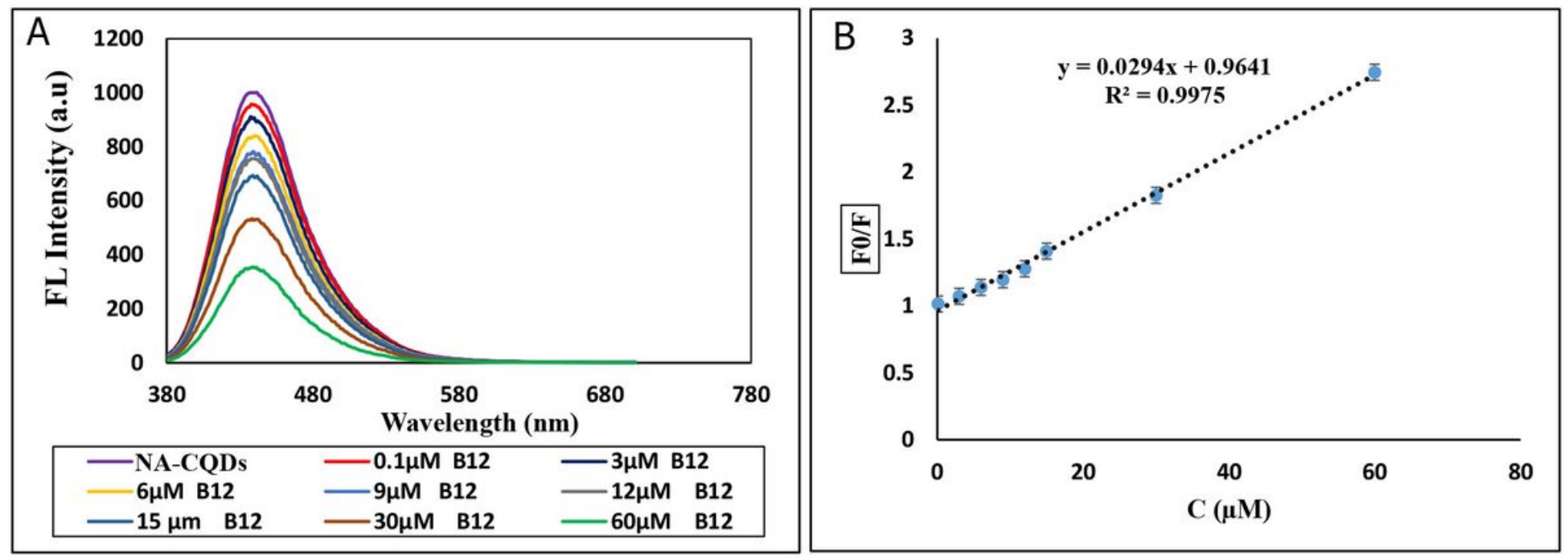

Figure 2

(A) Changes in fluorescent emission intensity of NA-CQDs upon successive addition of VB12. (B) Linear regression graph of F0/F values against VB12 concentration in the ranges of 0.1-60 $\mu \mathrm{M}$

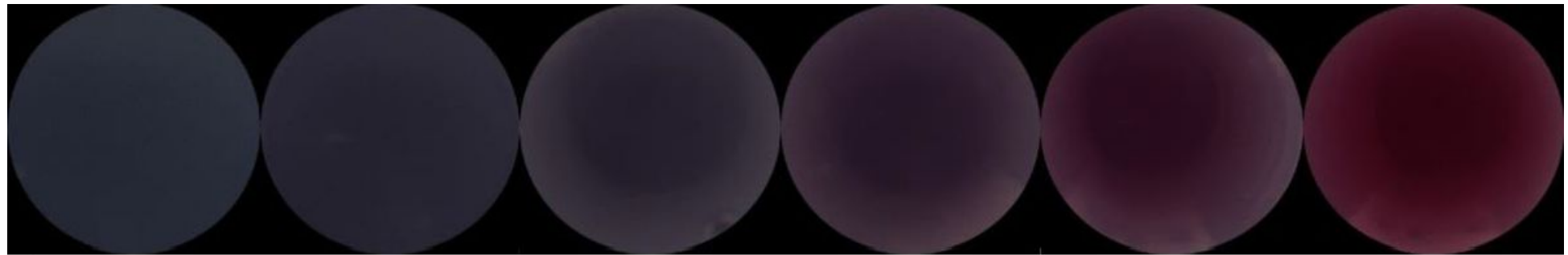

A

$\underset{0-66.6 \mu \mathrm{M}}{\longrightarrow}$

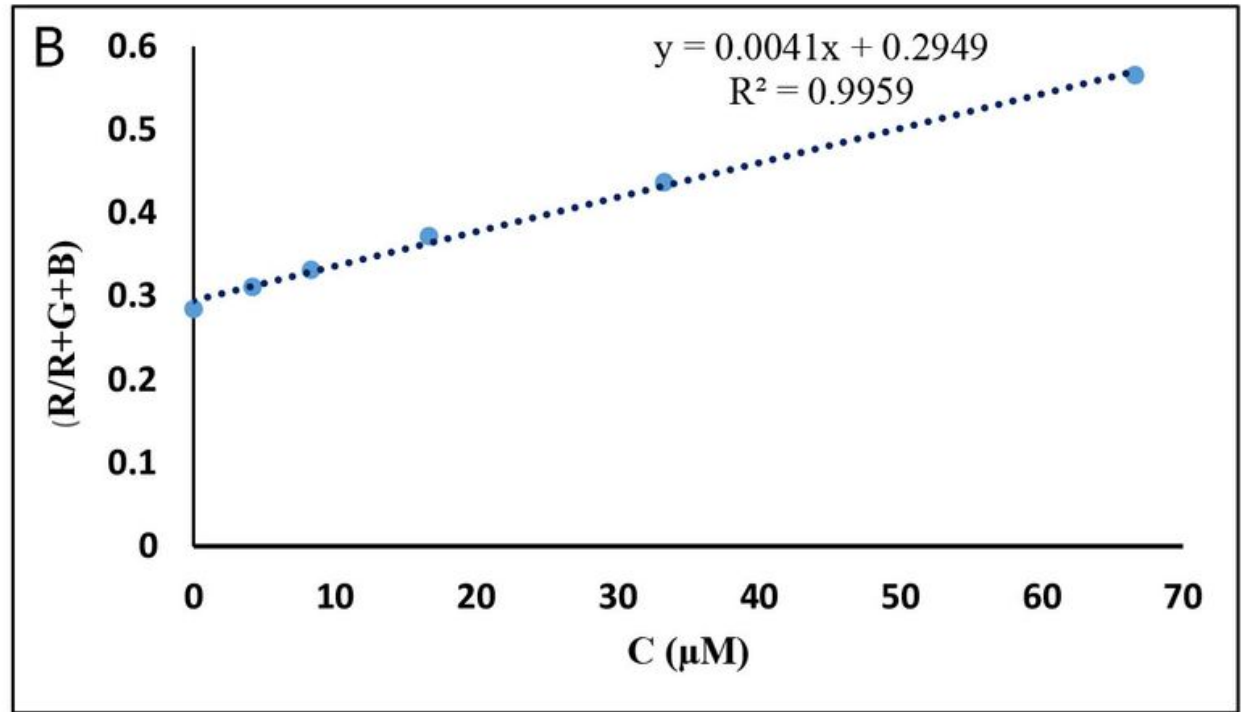

Figure 3

Smartphone-assisted colorimetric sensing platform on NA-CQDs. (A) Images of NA-CQDs upon successive addition of VB12, which were directly taken by the smartphone digital camera. (B) Linear regression graph of $(R) /(R+G+B)$ values against VB12 concentration in the ranges of 0-66.6 $\mu \mathrm{M}$ 

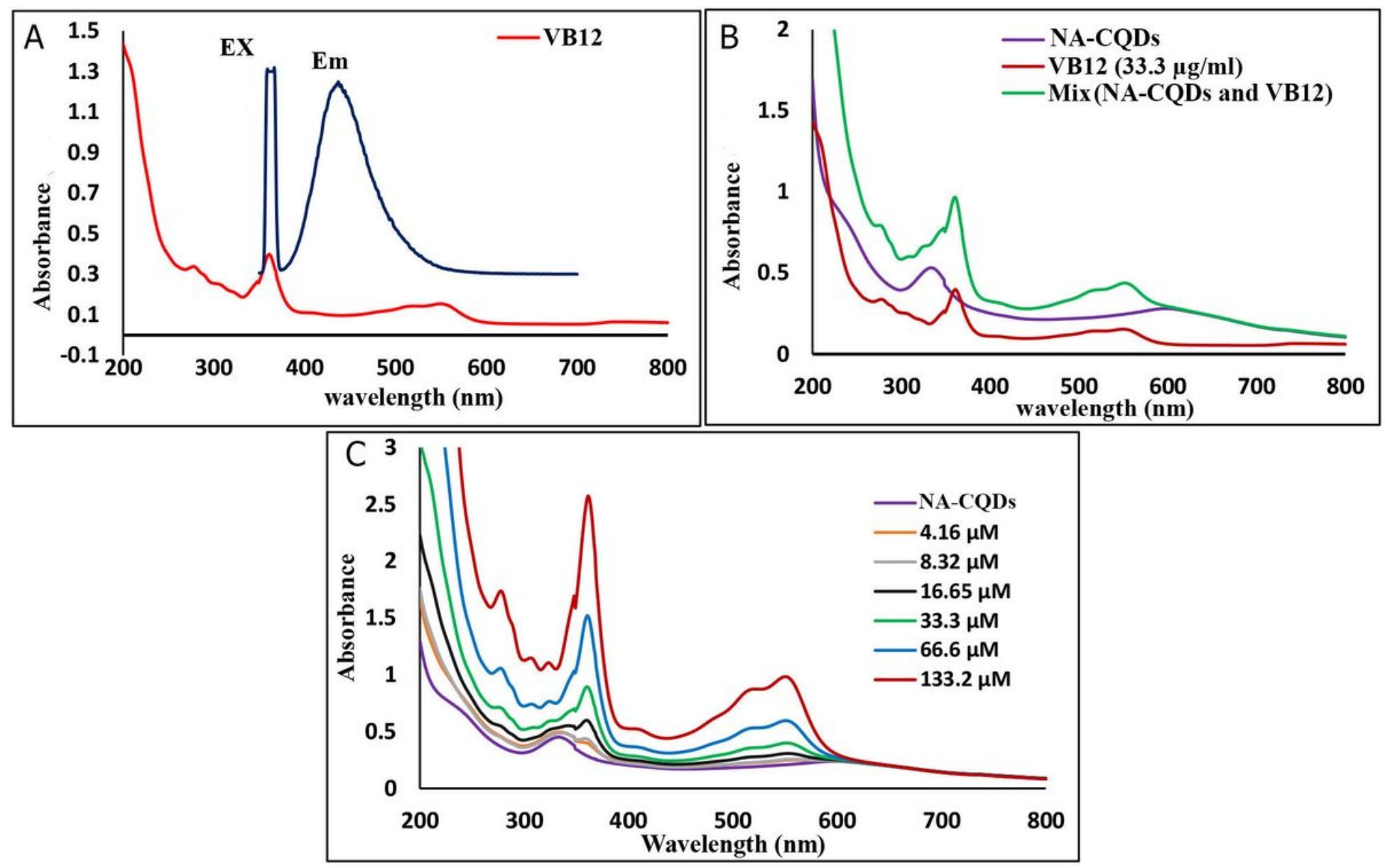

Figure 4

(A) UV-Vis absorption spectra of VB12 and fluorescent excitation and emission spectra of NA-CQDs nanosensor. (B) UV-Vis absorption spectra of free NA-CQDs, VB12 and mixture of NA-CQDs/VB12 sensing system. (C) UV-Vis absorption spectra of NA-CQDs nanosensor upon successive addition of VB12 in the ranges of 0-133.2 $\mu \mathrm{M}$ 

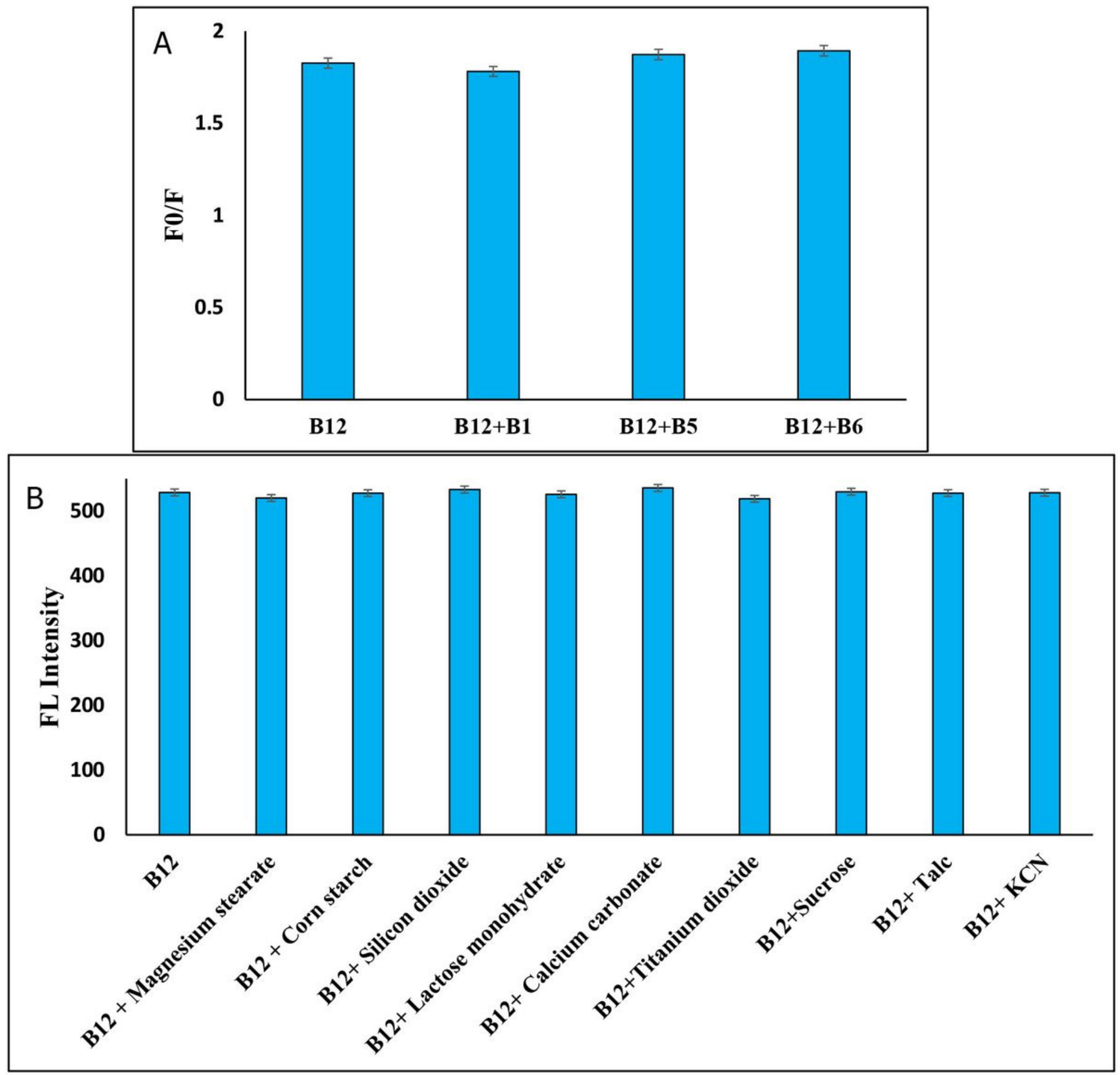

Figure 5

(A) Selectivity of the NA-CQDs nanosensor toward VB12 $(30 \mu \mathrm{M})$ in the presence of vitamin B1 $(3000 \mu \mathrm{M})$

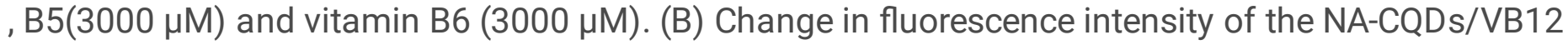
sensing system in the absence and presence of different coexistent excipients at pharmaceutical formulation.

\section{Supplementary Files}


This is a list of supplementary files associated with this preprint. Click to download.

- ESMJournaloffluorescence.doc 